\title{
Lipedematous scalp with lipedematous alopecia: A case report
}

\author{
Fariz Sarshar, Syed Suhail Amin, Mohammad Adil, Fatima Tuz Zahra \\ Jawaharlal Nehru Medical College (JNMC), Aligarh Muslim University (AMU), Aligarh, India \\ Corresponding author: Fatima Tuz Zahra, MD, E-mail: ftz0606@gmail.com
}

\begin{abstract}
Lipedematous scalp and lipedematous alopecia are a rare cutaneous disorders of unknown etiology characterised by thickening of subcutaneous fat layer presenting with thick, boggy scalp of spongy consistency. In addition to changes in texture of skin, varying degree of hair loss are seen in patients of lipedematous alopecia. It was first described by Cornbleet in 1935. This is the report of 40 years female who presented with asymptomatic boggy swelling over affected scalp for 5 months and headache for 1 year. The swelling was associated with patchy hair loss. Exact etiopathogenesis remains unclear. Lipedematous scalp can be a possible cause of dysaesthesia of scalp. Herein, we report a case of lipedematous alopecia due to its rare occurrence and classical presentation with emphasis on trichoscopic, histopathological and radiological findings.
\end{abstract}

Key words: Lipedematous scalp; Lipedematous alopecia; Lipomatosis

\section{INTRODUCTION}

Lipedematous scalp is a rare disorder of unknown etiology characterised by thickening of subcutaneous tissue which presents as thick and boggy scalp usually localised to vertex and parietal area. Lipedematous alopecia refers to the condition where hair growth abnormalities co-exist with lipedematous scalp. Lipedematous alopecia may be confused with other causes of localised hair loss such as alopecia areata [1]. Herein, we report a case of lipedematous scalp and alopecia present at the same site over the scalp.

\section{CASE REPORT}

A 40 years female presented with complaints of headache for 1 year and gradually progressive asymptomatic swelling over vertex of scalp noticed for 5 months. The swelling was insidious in onset and associated with single patch of hair loss for 3 months. Medical and family history was unremarkable. On inspection $2 * 3 \mathrm{~cm}$ solitary patch of non-scarring alopecia was present over vertex area with no scaling and erythema (Fig. 1). On palpation, scalp had a thick, soft and spongy consistency underneath the alopecia patch (Fig. 2). Hair pull test was negative. Trichoscopic examination revealed follicular plugging, white dots and variable erythema (Fig. 3). Ultrasonography (USG) of scalp showed well defined heterogeneous hyperechoic lesion with mild internal vascularity in subcutaneous plane of Bilateral Fronto-ParietoTemporal region suggestive of lipomatous lesion. Magnetic resonance imaging (MRI) scalp showed the thickness of $11.4 \mathrm{~mm}$ in the vertex region (Fig. 4). Punch biopsy of scalp showed increased subcutaneous tissue thickness with fibro-collagenous dermis showing hair follicle and sebaceous gland and mature adipose tissue encroaching the dermis (Fig. 5). Based on above findings diagnosis of lipedematous alopecia was made.

\section{DISCUSSION}

Lipedematous scalp was first described by Cornbleet in 1935 and Coskey et al. in 1961 first reported the case of lipedematous alopecia. The disease is more common 


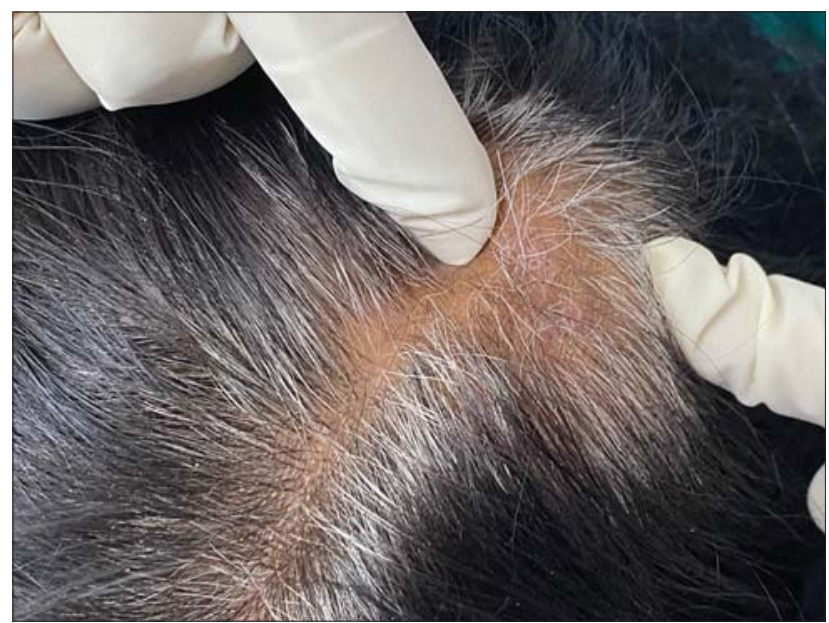

Figure 1: Patch of non-scarring alopecia present over vertex.

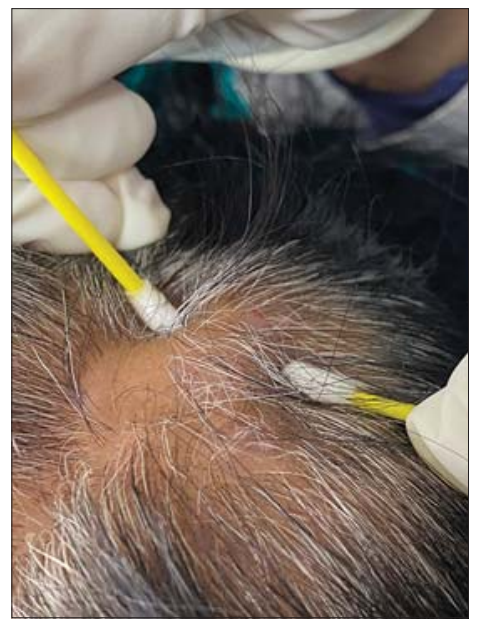

Figure 2: Boggy scalp underneath the alopecia patch.

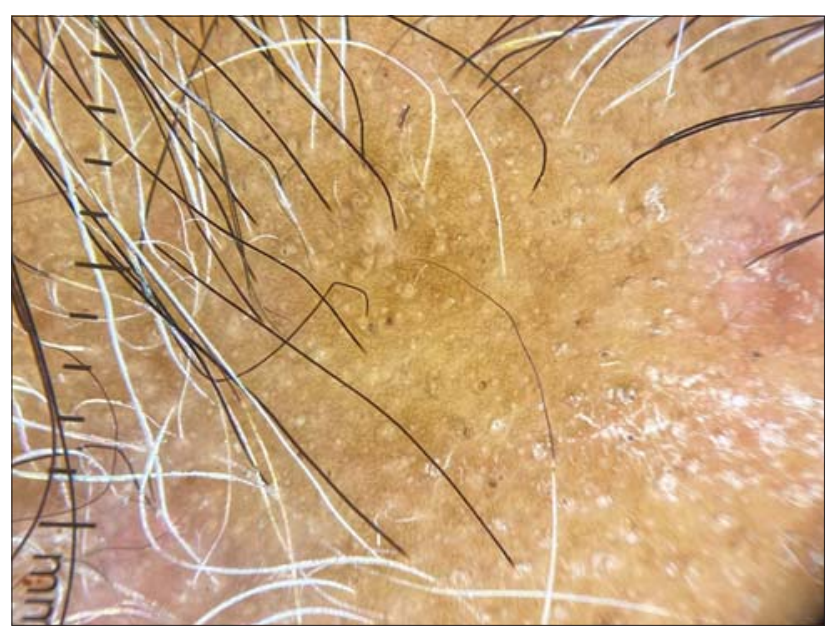

Figure 3: Dermoscopy showing follicular plugging, white dots and erythema.

in females (90\%) and in European, African American and Egyptian people [2]. It can begin at any age and in fact, congenital cases have been reported [3].

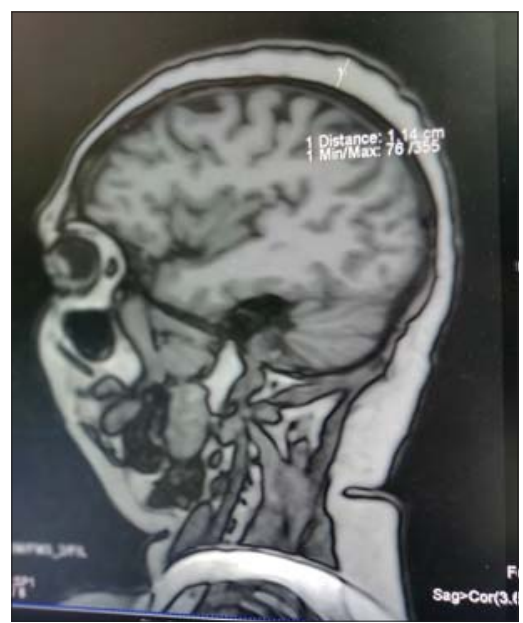

Figure 4: Magnetic Resonance Imaging showing increased thickness of scalp.

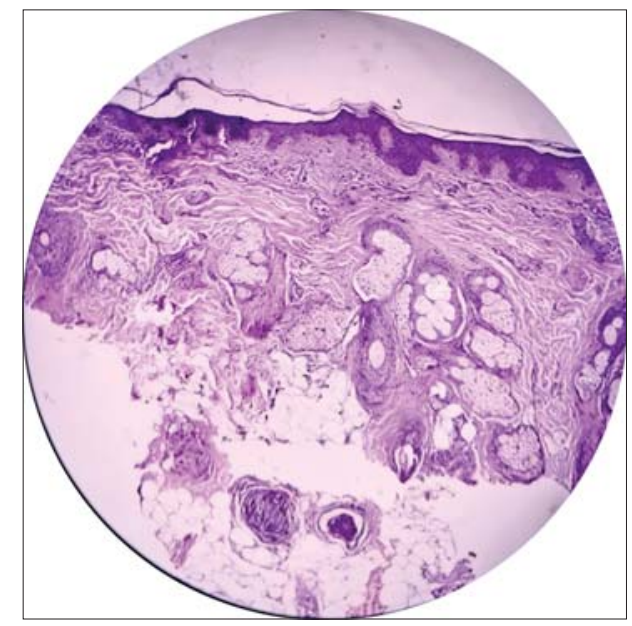

Figure 5: Histopathology showing increased subcutaneous tissue thickness with hair follicle, sebaceous gland and mature adipose tissue encroaching the dermis.

The disease presents as a boggy swelling of the scalp with predominant involvement of the vertex and occiput. Patient may also have associated symptoms of pain, paraesthesia, tenderness and pruritus. No definite cause has been established but role of leptin has been proposed which causes adipocytes hyperplasia and displacement of adipocytes into dermis [4]. Hormonal and genetic factors and use of tight head gear have been implicated [5]. Martin et al. proposed that thickened adipose tissue may cause pressure effect on hair follicle or impairment of nutrition of the hair matrix leading to decrease in hair growth or shortening of anagen phase [6]. This might be responsible for the broken hair and associated hair loss in areas corresponding to the soft, boggy scalp area. Lymphangiectasia has also been proposed to cause hair loss. Headache may result from pressure on dermal nerves caused by dermal oedema and thickening [7]. 
The diagnosis depends on clinical features \& histopathological findings. Subcutaneous tissue thickening with encroachment into the dermis, without inflammation is the chief histopathological feature [5]. MRI, CT and USG are used to measure increased scalp thickness. In India, the mean scalp thickness on MRI was found to range from 5.5 to 7.75 $\mathrm{mm}$ in lipedematous alopecia and from 9.2 to $16 \mathrm{~mm}$ in lipedematous scalp in different regions of the scalp [8].

Alittle over 50 cases of lipedematous scalp and lipedematous alopecia have been reported till now. The condition has been reported to co-exist with psoriasis, mucinosis, lupus erythematosus and androgenetic alopecia [2,5]. Sahu et al. reported concomitant alopecia areata and lipedematous scalp over parietotemporal region of scalp in a 29 year old male [7]. Dhurat et al. also described a similar case and emphasized that lipedematous scalp and lipedematous alopecia are not distinct conditions [1].

\section{CONCLUSION}

Till now no concrete and successful treatment option exists to treat lipedematous scalp. Intralesional steroids, mycophenolate mofetil and surgical debulking and scalp reduction have been tried. Our patient received a trial of intralesional triamcinolone injection. Follow-up showed no clinical signs of improvement. Given the limited cases of successful treatment, further clinical evidence is needed to reach a consensus for treatment of lipedematous alopecia.

\section{Consent}

The examination of the patient was conducted according to the principles of the Declaration of Helsinki.
The authors certify that they have obtained all appropriate patient consent forms, in which the patients gave their consent for images and other clinical information to be included in the journal. The patients understand that their names and initials will not be published and due effort will be made to conceal their identity, but that anonymity cannot be guaranteed.

\section{REFERENCES}

1. Dhurat RS, Daruwalla SB, Ghate SS, Jage MM, Sharma A. Distinguishing lipedematous scalp, lipedematous alopecia and diffuse alopecia areata. Skin Appendage Disord. 2019;5:316-9.

2. Müller CS, Niclou M, Vogt T, Pföhler C. Lipedematous diseases of the scalp are not separate entities but part of a spectrum of lipomatous lesions. J Dtsch Dermatol Ges. 2012;10:501-7.

3. Lee HE, Kim SJ, Im M, Kim CD, Seo YJ, and Lee JH, et al. Congenital lipedematous alopecia: Adding to the differential diagnosis of congenital alopecia. Ann Dermatol. 2015;27:87-9.

4. Yasar S, Gunes P, Serdar ZA, Tosun I. Clinical and pathological features of 31 cases of lipedematous scalp and lipedematous alopecia. Eur J Dermatol. 2011;21:520-8.

5. Carrasco-Zuber JE, Alvarez-Veliz S, Cataldo-Cerda K, Gonzalez-Bombardiere S. Lipedematous scalp: a case report and review of the current literature. J Dtsch Dermatol Ges. 2016;14:418-21.

6. Martín JM, Monteagudo C, Montesinos E, Guijarro J, Llombart B, Jordá E. Lipedematous scalp and lipedematous alopecia: a clinical and histologic analysis of 3 cases. J Am Acad Dermatol. 2005;52:152-6.

7. Sahu P, Sangal B, Dayal S, Kumar S. Lipedematous scalp with varied presentations: A case series of four patients. Indian Dermatol Online J. 2019;10:571-3.

8. Dincy Peter CV, Jennifer A, Raychaudhary T, Chandrashekhar L, Merilyn S, Gowda S, et al. Lipedematous scalp. Indian J Dermatol Venereol Leprol. 2014;80:270-2.

Copyright by Fariz Sarshar, et al. This is an open-access article distributed under the terms of the Creative Commons Attribution License, which permits unrestricted use, distribution, and reproduction in any medium, provided the original author and source are credited.

Source of Support: Nil, Conflict of Interest: None declared. 